\title{
Application of Risk-based Flexibility Assessment Methods to Evaluate System Expansion Plans
}

\author{
Baorong Zhou ${ }^{1}$, Pei Zhang ${ }^{2}$, Jianbin Chen ${ }^{1}$, Xiaoming Jin $^{1}$, Kai Hou ${ }^{2}$, Ming Niu ${ }^{3}$, \\ Zhao Xü ${ }^{3}$, Yunkai Lei ${ }^{2}$ \\ ${ }^{1}$ Electric Power Research Institute of China Southern Power Grid, Guangzhou, China \\ ${ }^{2}$ Tianjin University, Tianjin, China \\ ${ }^{3}$ Department of Electrical Engineering, The Hong Kong Polytechnic University, Hong Kong SAR, China \\ Email: brzhou@csg.cn, peizhang166@gmail.com;
}

Received April, 2013

\begin{abstract}
This paper proposed a flexibility assessment approach based on and risk assessment methodology. System planners prioritize each planning scheme with consideration of three aspects: reliability, economics, and flexibility. In the past, there is lack of quantitative indices to measure flexibility of a power system. This paper proposes applying probabilistic risk assessment method to quantify system flexibility. The proposed approach is demonstrated to compare two transmission planning schemes during Guangdong expansion planning process.
\end{abstract}

Keywords: Flexibility Assessment; Transmission Scheme Prioritization

\section{Introduction}

Development of power systems is of the foundations of the national economy. Thus power system planning is always one of the most important aspects of electric industry. In order to guarantee the reliability and economy of the power supply, as well as to meet the rapidly increasing demand of electric industry, power system planners generally establish several planning schemes, among which, only the most reliable, economical and flexible one will be implemented. Therefore, it is great importance for planners to apply reasonable methods to perform assessment and prioritization for those schemes.

Regarding assessment and prioritization, there are clear rules and effective methods currently to conduct reliability and economy assessment. However, there is no recognized standard or valid approach associate with the flexibility assessment. The flexibility of planning schemes indicates their tolerance for uncertain factors, major of which are listed as follows:

- Uncertainty of fault probability of components such as generator, line and transformer;

- Uncertainty of power generation location, capacity, timing, and availability of new generating facilities;

- Uncertainty of future demand, scope and complexity of transaction;

- Uncertainty of regulatory constraints and rules.

To handle these uncertainties, McCalley and his associates have developed a risk-based method for power system security assessment [1]. This method considers a predefined set of contingencies, and takes into account both probabilities and impacts of these events. The impacts are measured in terms of the voltage or current violations caused by these predefined contingencies. This innovative method has been successfully implemented in numerous fields such as network planning, operational planning and operation [2-5].

Based on the risk assessment theory, EPRI, together with some electric power corporations, has developed a Probabilistic Risk Assessment (PRA) methodology and further software to perform risk assessment on power systems [6]. The PRA program computes the probabilistic risk indices and displays the results clearly with forms and images. Taking into account both probabilities and physical impacts of contingencies, planners are able to have a better understanding of planning schemes, as well as identify potential failure modes. PRA methodology and software have been implemented in a number of electric power corporations, such as Southern Company, American Electric Power, KCP\&L, the Eastern Interconnection, NYPA, ERCOT and Tri-State. At present, PRA method is being introduced into over ten utilities for their own studies [7-9].

This paper proposes using probabilistic risk assessment method to perform flexibility assessment and quantify system flexibility. Quantitative indices can be used by system planners to prioritize planning schemes. The second part of this paper presents PRA methodology. 
The third part of this paper introduces two expansion planning schemes of Guangdong Power and study assumptions. The fourth part of this paper presents flexibility assessment results using PRA methods and prioritization based on comparison of risk indices.

\section{Risk-based Flexibility Assessment Method}

Flexibility assessment measures the capabilities of the designed power system to handle future uncertainties. As shown in Figure 1, system operators need to know where the potential problems are located, how likely they will happen, and how far the operating point is close to the boundaries [7]. Risk assessment should provide detail information of potential dangers, just like radars. The traditional deterministic contingency analysis is unable to catch the diverse probabilities of events that lead to potential security limit violations. PRA method takes both likelihood and impacts of each contingency into consideration, and work out a single reliability index - Probabilistic Risk Index (PRI). We proposed to use these quantitative risk indices to evaluate system flexibility.

\subsection{Probabilistic Risk Indices}

PRI is defined as the product of an impact by a probability.

$$
\text { PRI = Probability } \times \text { Impact }
$$

where the probability quantifies the likelihood of the simulated outage configuration, and the physical impact quantifies the severity of the situation.

\subsection{Impacts}

According to different types of impacts, there are four major risk indices: $I_{\mathrm{PRI}, \mathrm{N}}$ (voltage violation), $I_{\mathrm{PRI}, \mathrm{O}}$ (amperage or thermal overload), $I_{\mathrm{PRI}, \mathrm{V}}$ (voltage instability) and $I_{\mathrm{PRI}, \mathrm{L}}$ (load loss):

- Voltage Violation Index $I_{\mathrm{PRI}, \mathrm{N}}$ :

$$
I_{\mathrm{PRI}, \mathrm{N}}=\sum_{i \in S_{\mathrm{VN}}} P_{\mathrm{ACC}, \mathrm{i}} V_{\mathrm{IMP}, \mathrm{i}}
$$

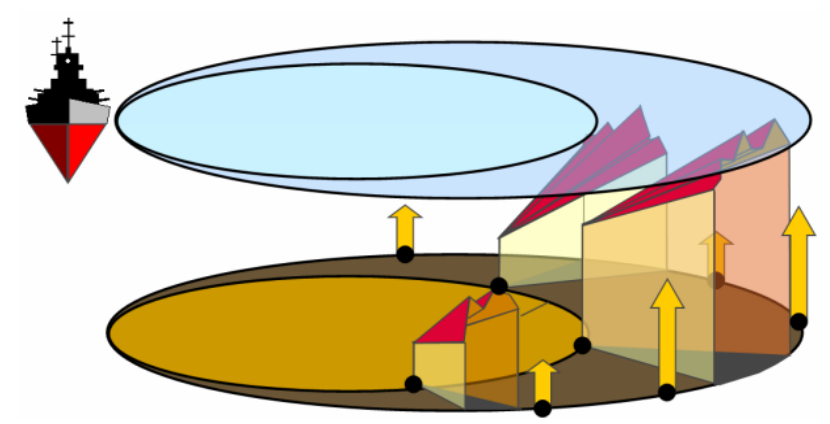

Figure 1. Operating power system is like navigating a ship [7]. where $V_{\mathrm{IMP}, \mathrm{i}}$ is the voltage violation from the bus upper or lower limits caused by the $i$ th contingency. The voltage impact is measured in terms of $\mathrm{kV}$ or p.u. $S_{\mathrm{VN}}$ is a set of contingencies that causes voltage violations.

- Thermal Overload Index IPRI,O:

$$
I_{\mathrm{PRI}, \mathrm{O}}=\sum_{i \in S_{\mathrm{LO}}} P_{\mathrm{ACC}, \mathrm{i}} A_{\mathrm{IMP}, \mathrm{i}}
$$

where $A_{\mathrm{IMP}, \mathrm{i}}$ is the thermal overload above the branch thermal rating caused by the $i$ th contingency. The thermal overload impact is measured in terms of MVA or KA. $S_{\mathrm{LO}}$ is a set of contingencies that cause overload.

- Voltage Instability Index IPRI,V:

$$
I_{\mathrm{PRI}, \mathrm{V}}=\sum_{i \in S_{\mathrm{VS}}} P_{\mathrm{ACC}, \mathrm{i}} V_{\mathrm{IMP}, \mathrm{Si}}
$$

where $V_{\mathrm{IMP}, \mathrm{Si}}$ is the voltage instability impact caused by the $i$ th critical situation. It is measured in binary format. If a situation causes the system becoming voltage unstable, the voltage stability impact value of is equal to 1 . Otherwise, it is equal to $0 . S_{\mathrm{Vs}}$ is a set of contingencies that cause voltage instability.

- Load Loss Index IPRI,L:

$$
I_{\mathrm{PRI}, \mathrm{L}}=\sum_{i \in S_{\mathrm{LR}}} P_{\mathrm{ACC}, \mathrm{i}} L_{\mathrm{IMP}, \mathrm{Li}}
$$

where $L_{\mathrm{IMP}, \mathrm{Li}}$ is the total load loss caused by the $i$ th situation. The load loss impact is measured in MW. $S_{\mathrm{LR}}$ is a set of contingencies that cause load loss.

\subsection{Probabilities}

In a planning context, probability is a measure of the likelihood that the power system will be in a given situation at a random time in the future, and is a function of the availability of every piece of equipment in the power system.

$$
\text { probability }=\prod_{i \in U} u\left(c_{\mathrm{i}}\right) \prod_{j \in A}\left(1-u\left(c_{\mathrm{j}}\right)\right)
$$

where $U$ represents the set of unavailable components; $A$ represents the set of available components; $\Omega=U \cup A, \Omega$ represents the complete set of system components. $u\left(c_{\mathrm{i}}\right)$ and $\left(1-u\left(c_{\mathrm{i}}\right)\right)$ represent the unavailability and availability rates of the component $i$, respectively.

\section{Study Data and Assumption}

In 2030, the total peak load demands of Guangdong electric power network will be approximately 175 MW, among which the load demands of Pearl River Delta will account for about $74 \%$. Outer area power transfer into Guangdong electric power network will be about 53.3 MW accounting for about $28 \%$ of the total load demands. $86 \%$ of 53.3MW will be transferred by HVDC. There are two expansion planning schemes for 2030 Guangdong electric power network frame. 
The first scheme succeeds the existing 500KV double looped network structure, as shown in Figure 2. The eastern and western parts of Guangdong electric power network are connected by two $500 \mathrm{kV}$ tie-lines which enable 2000MW power transfer. This system includes 7972 buses, 6370 lines and 5736 transformers.

The second scheme, as shown in Figure 3, plans to build a 1000KV UHV transmission network to connect major nuclear power plants that are located at eastern and western parts of Guangdong province. This system consists of 7886 buses, 6349 lines and 5668 transformers.

Contingency analyses of the two planning schemes were performed using BPA software. The contingency analysis results provide the detailed information of physical impact of each contingency.

The unavailability rate of a line is estimated using the following equation:

$$
u=\text { Outage Freq } \times \text { Repair Time } / 8760
$$

The outage frequency is estimated using the following equation:

$$
\text { Outage Freq }=a+b \times(Z / \text { ZpuPerMile })
$$

where $a$ (1/year) is the constant parameter of the forced outage frequency; $b$ (1/year/mile) is the proportional parameter of the forced outage frequency; ZpuPerMile (pu/mile) is the average impedance (p.u.) per mile used to estimate the line length; RepairTime (hour) is the average repair time (hour) after a forced outage.

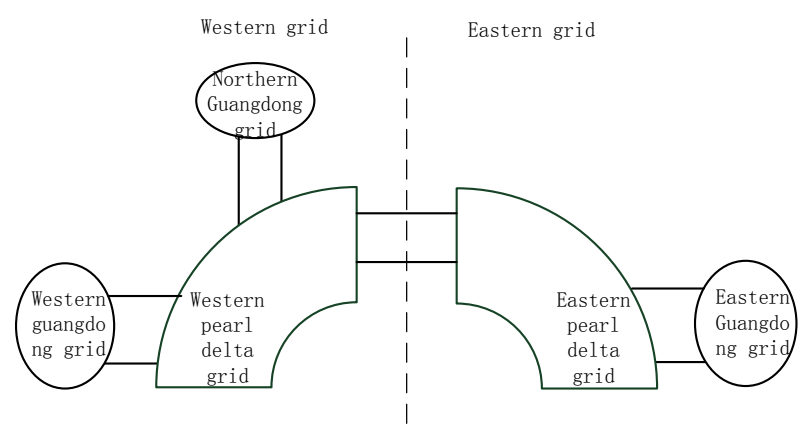

Figure 2. High Voltage DC Transmission Scheme.

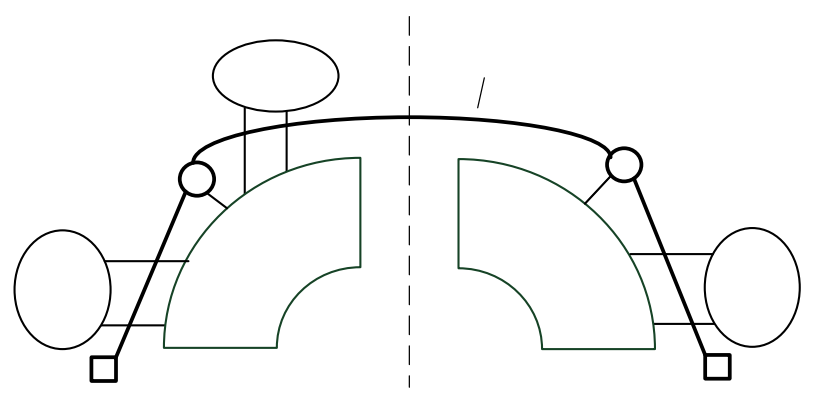

Figure 3. Ultra high voltage DC transmission scheme.

\section{Flexibility Assessment and Prioritization of Planning Schemes Using PRA Method}

We performed risk-based flexibility assessment for two planning schemes and calculate quantitative risk indices, which are used as flexibility indices of planning schemes. Prioritization can be performed by system planners based on these indices.

Table 1 provides a summary of risk indices for two planning schemes. With consideration of four risk factors: overload, voltage and voltage instability, and load loss, it clearly shows that Scheme A has lower risk comparing with Scheme B. This indicates Scheme A has a better flexibility than Scheme B.

Figure 4 show the risk analysis of bus voltage violations, where it is shown that the risk indices of schemes $\mathrm{A}$ and $\mathrm{B}$ are 2.623E-5 and 1.799E-3 respectively. Obviously, the risk of scheme B is larger than that of scheme A more than 60 times from a voltage violation risk perspective.

Figure 5 shows the risk analysis of transmission line overloading, where it is shown that the risk indices of schemes A and B are 9.778 and 10.928 respectively. Obviously, the risk of scheme $\mathrm{A}$ is larger than that of scheme $\mathrm{B}$ from the overload perspective, because the overloading risk of scheme B is 1.12 times of scheme A.

Figure 6 shows the risk analysis of voltage instability, where it is shown that the risk indices of schemes $\mathrm{A}$ and $\mathrm{B}$ are 7.379E-3 and 3.014E-2 respectively. From voltage instability perspective, scheme A has higher risk of voltage instability than Scheme B.

Table 1. Overall Risk-baesed Felxibility Analysis ResulT.

\begin{tabular}{lcccc}
\hline & $\begin{array}{c}\text { Voltage } \\
\text { violation }\end{array}$ & Overload & $\begin{array}{c}\text { Voltage } \\
\text { instability }\end{array}$ & Load loss \\
\hline Scheme A & $2.623 \mathrm{E}-5$ & 9.778 & $7.379 \mathrm{E}-3$ & 0 \\
Scheme B & $1.799 \mathrm{E}-3$ & 10.928 & $3.014 \mathrm{E}-2$ & 0 \\
\hline
\end{tabular}

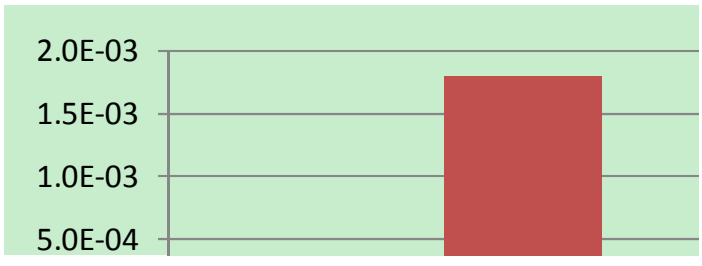

Figure 4. Risk analysis of bus voltage violations.

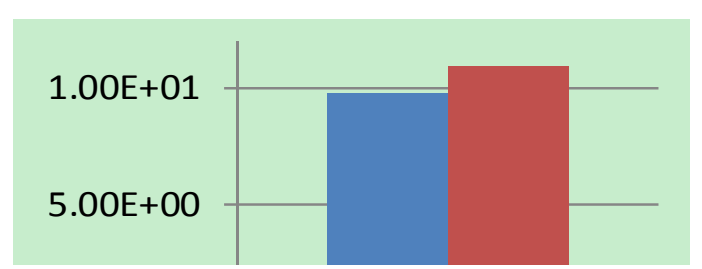

Figure 5. Risk Analysis of line overloading. 


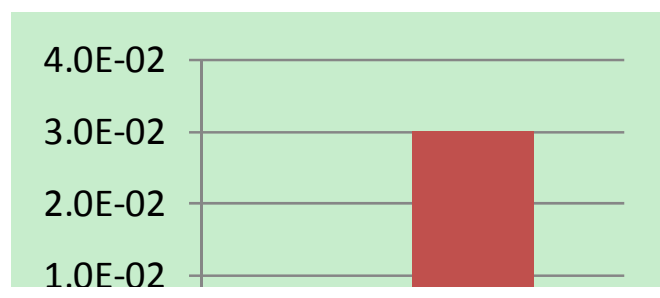

Figure 6. Risk analysis of voltage instability.

Considering the results above, scheme A obviously outperforms scheme B in all three aspects. Therefore it can be concluded that according to risk-based flexibility analysis, scheme A is a better flexibility and is recommended to be adopted by the utility.

\section{Conclusions}

This paper introduced a new risk-based flexibility assessment and prioritization method for planning schemes. Two planning schemes of Guangdong electric power network were studied and the risk-based flexibility indices were computed, including voltage violation index, thermal overload index, voltage instability index and load loss index. With this flexibility information, system planners can evaluate the flexibility and prioritize those project schemes. The flexibility assessment helps system planners evaluate the operational risks when designing the future power system, therefore builds a linkage between system planning and system operation.

\section{REFERENCES}

[1] J. D. McCalley, V. Vittal and N. Abi-Samra, "An Overview of Risk Based Security Assessment," in Power Engineering Society Summer Meeting, 1999. IEEE, Vol. 1, 1999, pp. 173-178.
[2] H. Wan, J. D. McCalley and V. Vittal, "Risk Based Voltage Security Assessment," Ieee Transactions on Power Systems, Vol. 15, 2000, pp. 1247-1254. doi:10.1109/59.898097

[3] Y. J. Dai, J. D. McCalley, N. Abi-Samra and V. Vittal, “Annual Risk Assessment for Overload Security,” Ieee Transactions on Power Systems, Vol. 16, 2001, pp. 616-623. doi:10.1109/59.962405

[4] W. H. Fu, J. D. McCalley and V. Vittal, "Risk Assessment for Transformer Loading," Ieee Transactions on Power Systems, Vol. 16, 2001, pp. 346-353. doi:10.1109/59.932267

[5] M. Ni, J. D. McCalley, V. Vittal and T. Tayyib, "Online Risk-based Security Assessment," Ieee Transactions on Power Systems, Vol. 18, 2003, pp. 258-265. doi:10.1109/TPWRS.2002.807091

[6] Z. Pei, S. T. Lee and D. Sobajic, "Moving Toward Probabilistic Reliability Assessment Methods," in Probabilistic Methods Applied to Power Systems, 2004 International Conference on, 2004, pp. 906-913.

[7] Z. Pei, M. Liang, L. Hopkins and B. Fardanesh, "Utility Experience Performing Probabilistic Risk Assessment for Operational Planning," in Intelligent Systems Applications to Power Systems, 2007. ISAP 2007. International Conference on, 2007, pp. 1-6.

[8] Z. Pei, M. Graham and D. Ramsay, "Prioritization of Transmission Projects using EPRI Probabilistic Risk Assessment Program,” in Power and Energy Engineering Conference, 2009. APPEEC 2009. Asia-Pacific, 2009, pp. 1-5.

[9] Z. Pei, L. Shanshan, X. Ruilin, L. Xinyu and F. Li, “Assessing System Risk and Integrating Operation and Planning Functions for Chongqing Power using EPRI Probabilistic Risk Assessment Program," in Power and Energy Engineering Conference (APPEEC), 2010 Asia-Pacific, 2010, pp. 1-6. 